\title{
Sutureless patch-and-glue technique for the repair of coronary sinus injuries
}

\author{
Marco Agrifoglio, MD, PhD, ${ }^{a}$ Fabio Barili, MD, Samer Kassem, MD, ${ }^{a}$ Luca Dainese, MD, ${ }^{a}$ Alessandro Parolari, MD, PhD, \\ Gianluca Pontone, MD, ${ }^{\mathrm{b}}$ Gianluca Polvani, MD, ${ }^{\mathrm{a}}$ Francesco Alamanni, MD, ${ }^{\mathrm{a}}$ and Paolo Biglioli, MD, ${ }^{\mathrm{a}}$ Milan, Italy
}

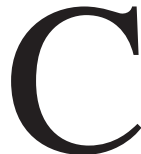

oronary sinus (CS) injury is a rare complication of CS catheter insertion. Its management can represent a technical challenge, leading to potential mortality. Several different surgical techniques have been proposed to repair CS injury, including pericardial or saphenous vein patches, ${ }^{1-4}$ because the primary suture of the CS often leads to stricture.

We report 7 cases in which CS injury was repaired with a sutureless patch-and-glue technique.

\section{Clinical Summary}

From January 1999 through November 2006, 7 cases of CS catheterrelated injuries occurred $(0.09 \%$ of cardiac operations with cardiopulmonary bypass in our experience). The patients were $1 \mathrm{man}$ and 6 women (mean age, $69 \pm 12$ years) who underwent valve replacement, coronary artery bypass grafting, or both during cardiopulmonary bypass. One patient had a reoperation.

After arterial and venous cannulation, a CS catheter was placed for retrograde delivery of cardioplegia. The same CS cannula was used in all cases $(14 \mathrm{~F} \times 27 \mathrm{~cm}$; Edwards Lifescience, Irvine, Calif), with the same infusion protocol $(150 \mathrm{~mL} / \mathrm{min}$, with $\mathrm{CS}$ pressures never exceeding $40 \mathrm{~mm} \mathrm{Hg}$ ).

All CS injures were intraoperatively detected at the time of blood cardioplegia delivery because an unexpected bleeding was observed. The delivery was stopped, and the heart was arrested with the anterograde delivery of cardioplegia. Direct inspection revealed a middle injury in all cases. The CS injury was repaired before performing cardiac surgery.

An autologous pericardium patch was sized to be about 4 times greater than the CS. It was fixed to the surrounding epicardium closed to the CS injury with two 4-0 Prolene stitches (Ethicon, Somerville, NJ). The surgical field was carefully kept dry with an air blower. A biologic bovine serum albumin and glutaraldehyde glue (Bioglue; CryoLife International, Inc, Kennesaw, Ga) was applied around the CS injury, starting from the atrial wall and extending to the ventricular

From the Departments of Cardiac Surgery ${ }^{\mathrm{a}}$ and Cardiology and Radiology, ${ }^{\mathrm{b}}$ Centro Cardiologico Monzino, University of Milan, Milan, Italy.

Received for publication Feb 8, 2007; revisions received March 15, 2007; accepted for publication April 19, 2007.

Address for reprints: Fabio Barili, MD, Department of Cardiac Surgery, University of Milan, Centro Cardiologico Monzino IRCCS, Via Parea 4, 20138 Milan, Italy (E-mail: fabarili@libero.it and fabio.barili@ unimi.it).

J Thorac Cardiovasc Surg 2007;134:522-3

$0022-5223 / \$ 32.00$

Copyright @ 2007 by The American Association for Thoracic Surgery

doi:10.1016/j.jtcvs.2007.04.012 wall. Perfect adherence among epicardium, glue, and patch was essential to avoid inhomogeneous distribution of the glue or lack of tension of the patch (Figure 1). The CS repair was tested with the delivery of anterograde cardioplegia, and when an optimal repair was observed, the planned cardiac operation was performed. The surgical procedure was complete without major technical difficulties in all 7 patients.

In our experience this simple surgical approach led to CS injury repair and a complete hemostasis. No perioperative complications occurred. The postoperative course was uneventful. The mean postoperative in-hospital stay was $7.2 \pm 2.1$ days. At follow-up, no death or cardiac complications occurred, and the multislice computed tomographic visualization of the heart showed the patency of the CS and the absence of CS strictures in all patients (Figure 2).

\section{Discussion}

The use of retrograde cardioplegia is a well-established technique that provides excellent myocardial protection with low complication rates. Although catheter-related CS injury is a rare event, ranging from $0.053 \%$ to $0.095 \%,{ }^{1-4}$ it can significantly worsen the operative and perioperative prognosis.

CS injury repair should be carefully performed to obtain complete hemostasis and a good venous drainage through the coronary veins. Several different techniques have been proposed because the simple direct suture of the injured CS edges can be challenging and lead to stricture and distortion, the bases of CS thrombosis. In our experience we adopted a modification of a previously described technique that involves a doublepatch technique sandwiched with human fibrin glue. ${ }^{3}$ In these cases the direct suture of a patch was avoided because the injury's edges were not clearly identified. No running sutures were used to secure the patch for the presence of a large hematoma and fragile tissues, and only 4 stay stitches avoided the patch's dislocation.

The effectiveness of the sutureless pericardial patch-and-glue technique for the repair of CS injuries is strictly related to the technical improvements in surgical adhesive and gluing substances. Bioglue has already demonstrated its facility in strengthening friable tissues and ensuring watertight sutures in surgical intervention in acute dissection of the aorta. ${ }^{5}$ It polymerizes rapidly, has elastic features, and effectively glues apposing tissues, allowing a complete hemostasis without increased tension and distortion.

This limited experience with the sutureless pericardial patchand-glue technique for repair of CS injuries indicates that it is safe and feasible for repair of central CS injuries. 

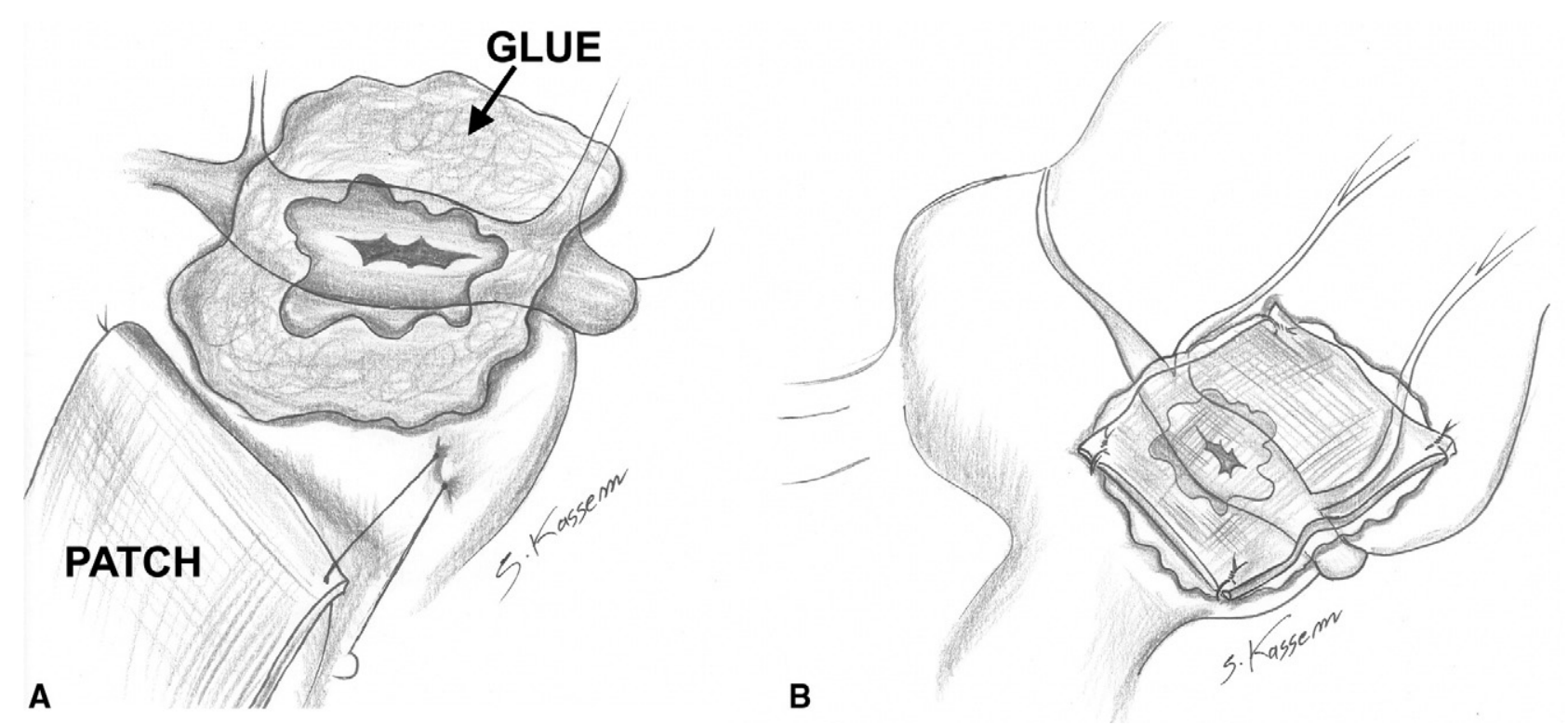

Figure 1. Performed repair of the coronary sinus injury with a sutureless pericardial patch-and-glue technique. After the identification of coronary sinus injury, a pericardial patch was fixed with 2 stitches to the surrounding atrial pericardium (A). The biologic glue was applied around the coronary sinus injury starting from the atrial wall and extending to the ventricular wall, and the patch was then fixed above the injury with another 2 stitches to avoid patch dislocation (B).

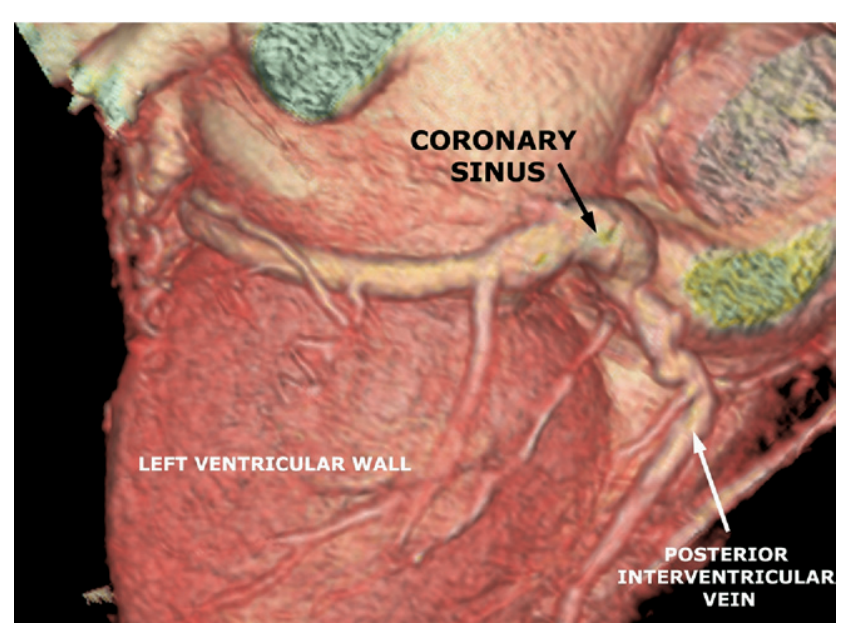

Figure 2. Multislice computed tomographic 3-dimensional reconstruction showing the coronary sinus patency and the absence of coronary sinus strictures. By its nature, the multislice computed tomography permitted us to clearly visualize the coronary sinus blood flow but not the coronary sinus patch.

\section{References}

1. Economopoulos GC, Michalis A, Palatianos GM, Sarris GE. Management of catheter-related injuries to the coronary sinus. Ann Thorac Surg. 2003;76:112-6.

2. Aharinejad S, Baumgartner H, Miksovsky A, Mohl W. Closure of ruptured coronary sinus by a pericardial patch. Ann Thorac Surg. 1996;62:889-91.

3. Aigner C, Wolner E, Mohl W. Management of central coronary sinus ruptures using the pericardial patch repair technique. Ann Thorac Surg. 2006;81:1275-8

4. Weiss SJ. Management of difficult coronary sinus ruptures. Ann Thorac Surg. 1994;58:548-50.

5. Fink D, Klein JJ, Kang H, Ergin MA. Application of biological glue in repair of intracardiac structural defects. Ann Thorac Surg. 2004; 77:506-11. 